\title{
PHOTOELECTRON SPECTROSCOPY OF MOLECULAR BEAMS
}

by J. Berkowitz

\section{NOTICE}

This report was prepared as an account of work sponsored by the United States Government. Neither the United States nor the United States Atomic Energy Commission, nor any of their employees, nor any of their contractors, subcontractors, or their employees, makes any warranty, express or implied, or assumes any legal liability of responsibility for the accuracy, completeness or usefulness of any information, apparatus, product or process disclosed, or represents that its use would not infringe privately owned rights.

\section{Presented at:}

NATO-Advanced Study Institute on Chemical Spectroscopy and Photochemistry in the Vacuum U1traviolet," August 5-17, 1973, Valmorin, Quebec, Canada

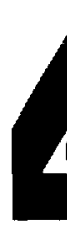


NATO-Advanced Study Institute on "Chemical Spectroscopy and Photochemistry in the Vacuum Ultraviolet", August 5-17, 1973, Valmorin, Quebec, Canada

PHOTOELECTRON SPECTROSCOPY OF MOLECULAR BEAMS*

\section{J. Berkowitz}

Argonne National Laboratory

Argonne, Illinois 60439

\section{INTRODUCTION AND CHEMICAL STUDIES}

The history of physical science in this century is replete with examples of phenomena initially discovered and investigated by physicists, which have subsequently become tools of the chemists. Visible and infrared spectroscopy, mass spectroscopy, nuclear magnetic resonance and Mössbauer experiments are but a few examples of this trend. I shall try to demonstrate in this paper that the field of photoelectron spectroscopy may develop in a reverse fashion.

Somewhat more than a decade ago, Vilesov, Kurbatov and Terenin (1) reported on the kinetic energy spectrum of electrons observed when monochromatized electromagnetic radiation with energy between 6 and $11.7 \mathrm{eV}$ was incident upon aromatic amiiles and methyl derivatives of benzene. This early study was limited in its upper energy by the absorption cutoff of LiF windows. About a year later, Turner and Al-Joboury (2) presented the first in a series of papers in which the resonance line of helium $(21.217 \mathrm{eV})$ was used as incident radiation, and analysis of photoelectrons was performed with a concentric cylindrical retarding field.

The introduction of the He(I) line turned out to be highly advantageous, not only because it can be generated as a sharp line with high intensity, but also because the phototonization cross sections of many common gases are near their maximum (3) at this wavelength. In addition, with $21.2 \mathrm{eV}$ one has sufficient energy to eject electrons from the valence orbitals of most compounds. This fortuitous confluence of circumstances strongly influenced the development of molecular photoelectron spectroscopy in the 
succeeding decade. The energy analyzers have improved during this period, but most studies still emphasize the measurement of ionization potentials with the $H e(I)$ line, and interpret their results in terms of the bonding (or antibonding) nature of the orbitals from which the corresponding electrons have been removed. A convenient sumary of such data 18 the handbook by Turner, Baker, Baker and Brundle (4). Other laboratories (5) that have contributed prominently to the measuresent of $584 \lambda$ photoelcetron spectra of volstile molecules include those of Price, Orchard, Hellbronner, Schwelg and Schaefer, Bock, Beck, Carlson, Frost and McDowell, Lindholm and others. Today the apparatus for such studies 1s commercially avallable and wore exotic and complex wolecules are continually being added to our store of 534 i photoelectron spectra.

Some molecules cannot readily be preparad as amblent gases in the steady state, and must be prepared as nolecular beans. This class of systens includes free radicals, excited atates of wolecules, atoms (besides the rare gases and mercury) and in general, anterials of low volutility.

The study of free radicals in beans has been pursued in the past few years at Southampton by Jonathan (6) and co-workers, who have detected $\mathrm{O}, \mathrm{N}, \mathrm{H}, \mathrm{O}_{2} \perp_{\Delta_{2}}$. CS; at Vancouver by Frost, McDowell (7) and collaboratsrs, who have reported on $\mathrm{HF}_{2}, \mathrm{ClO}_{2}$, $\mathrm{S}_{2} \mathrm{O}, \mathrm{O}_{3}$ and also CS; and recently at Sussex by Kroto (8) and co-workers.

Relatively involatile systems had been investigated at Argonne by Dr. J. L. Dehwer and the author (9). For this purpose, an apparatus of high collection efficlency was conetructed. In initial studies on the thalliun halides, it was found that electrons localized on the halogen, which acted as nonbonding lone pairs in the case of the relativaly covalent hydrogen halides and wathyl halides, were the bonding ciectrone in the ionic thallien halides. Thus, if the thallium halide in its ground state is closely approxinated by the representation $\pi 1^{+x^{-}}$, then

$$
\mathrm{TI} \mathrm{X}^{-}+\mathrm{hv}+\mathrm{T} 1^{+\mathrm{X}^{0}}+\mathrm{e}
$$

arcurs when the electron is rewoved from the halogen-11ke orbital. If tite Initial bonding had strong contribution from the coulombic $e^{2} / r$ potential, which is of the order of $-5 \mathrm{eV}$, chis is drastically reduciad in the ion to $-\alpha e^{2} / 2 x^{4}$, which is of order 0.2 eV. By contrast, an electron rewoved from a netal-like orbital can be represented by

$$
\mathrm{T} 1^{+\mathrm{X}^{-}}+\mathrm{hv}+\mathrm{T}^{+4 \mathrm{X}} \mathrm{C}^{-}+\mathrm{e}^{-} \text {. }
$$

resulting in an lonic state wore strongly bound than the ncutral ground state. This behavior has since been verified in the indium halides, and by extensive ab initio calculations as well as 
sentempirical calculations on the aluminum and galliun halides. Hence, the extension of photoelectron spectroscopy to the etudy of high tenperature spectes has proved rewarding by providing a drametc Illuatration of the different bonding characteristics of Ionic wolecules (which tend to be involatile) and covalent wolecules (which tend to be volatile).

We have recently extended these studies to the very Ionic alkali halldes. There 18 , of course, a large transfer of charge occurring in the formation of $\mathrm{M}^{+} \mathrm{X}^{-}$from $\mathrm{y}^{\circ}$ and $\mathrm{X}^{0}$. The consequence if a filled halogen p-like nolecular orbital, while the first metal-like orbital 1s the next inner p shell (for all cases except 1tthium). Each of these p-type orbitals can be expected to be eplit by the cylindrical molecular field into a pr and po orbital, the $n \pi$ being the less bound one on the halogen end, the po on the metal end ince the spliteing is caused by potentials of opposite etgn. The Ionteation potential of the halogen-1ike orbital should be roughly the sum of the electron affinity of the halogen negacive ion and ite bindiny energy in the field of the positive ion, or ca. $3 \mathrm{eV}+5 \circ \mathrm{V}=\mathrm{s} \mathrm{eV}$. As in the thallium, Indium and gal12um wonohalides, we anticipate that rewoval of an electron from an orbital localized around the halogen will severely dierupt the Ionic molecular bond.

The Ionization of the matal p-11ke orbital should occur roughly at the same energy as it does in the fres atom, nodified by a chenical shift due to charge transfer of the valence selectron, not unlike the chentcal shift obeerved in BSCA atudies. Th1s matal p ionization energy to -38 oV for sodium, -25 eV for potanelua, $-20-22$ eV for rubidium and $-17-19$ eV for cestum. Hence, with an Ionizing photon energy of $21.2 \mathrm{eV}$, the only wetal p-type orbitals likely to be observed would be in the cesiu halldel:.

The $584 \boldsymbol{\lambda}$ photoelectron spectru of cesiu lodide is shown in 15. 1. We note the two reglons of Ionic states, the one around 8 eV corresponding to Iontsation from the lodide 5 p-like orbital, the other around $19 \mathrm{eV}$ corresponding to loniation from the cesium Sp-1ike orbital. Walker (10), Fither and I have exanined the aplitting of each of these orbitals in some detail. In addition to the aforementioned $=0$ eplittins, which seens to scale inversely with the ionicity of the nolecule, there exists apin-orbit splitting wich has very nearly the same apin-orbit coupling parameter as the corxesponding atom. That 1s, the lodide Sp-like orbital has a spin-orbit coupling paraneter close to that of atomic lodine 5p. From ab initio calculations avallable for 9 of the 20 alkali halides and extrapolations of these results, we have been able to construct a table of $\pi-0$ oplittings for the outermost, halogen-1ike orbitals of alt of the alkalt halides, and also of their epin-orbit eplittings. These two types of splitting are of 


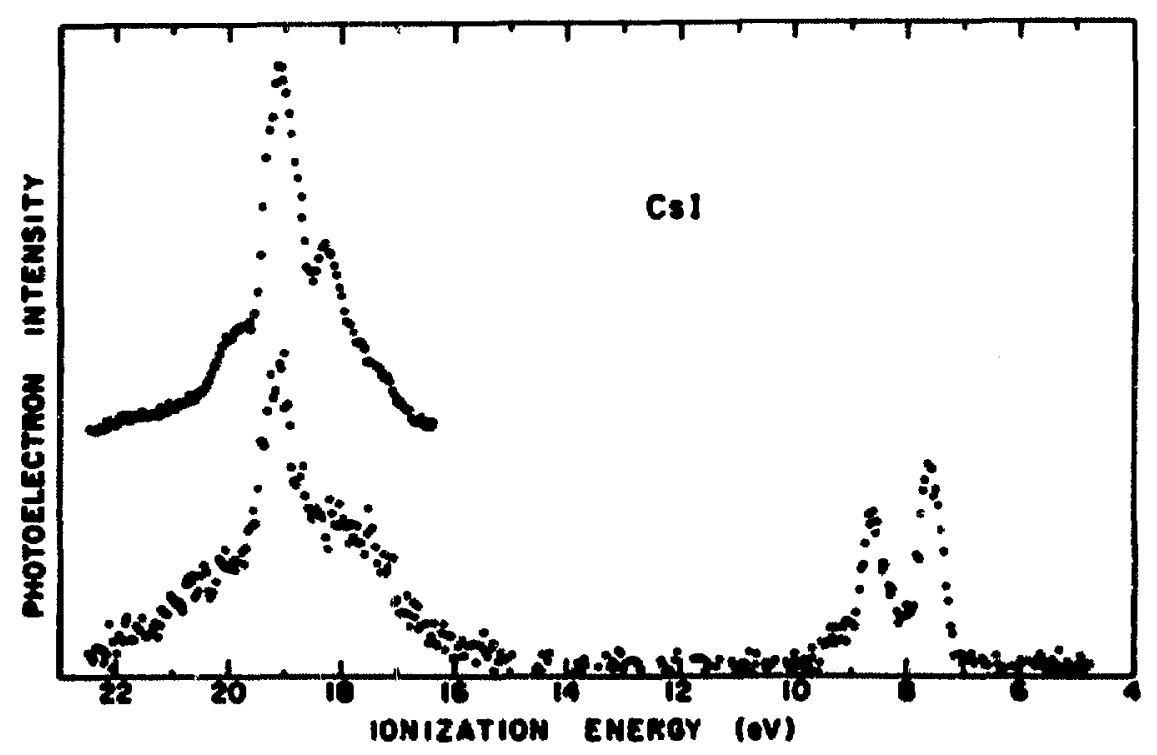

F18. 1. The He(I) photoelectron epectrun of CeI.

comparable magnitude, and ultimately it is necessary to conetruct and solve $2 \times 2$ secular equation to deduce the resulting states. For example, the lodide Sp-ilke orbital of cestum lodide has a i-o spliteing of $-0.12 \mathrm{eV}$ and apin orbic eplitting of $0.63 \mathrm{eV}$. The initial oplitting diagran is shown fn Fig. 2 . The $2 \pi_{1 / 2}$ and $2 \varepsilon_{1 / 2}$ state, having the same $\Omega$ value, then interact, and the magnitude of

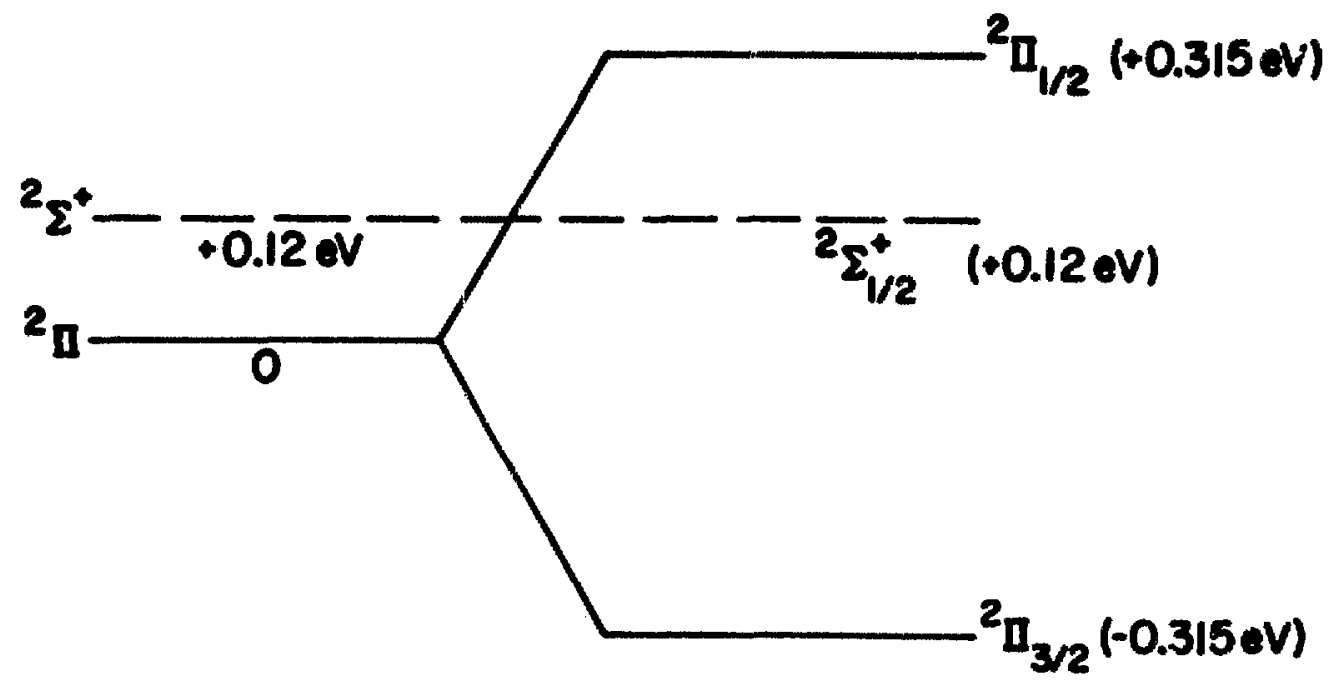

F18. 2. Partial splitting diagran of the CsI (iodide orbital) Ionlzation, Including $\pi-\sigma$ and spin-orbit effects. 
their repulsion can be obtained from the solution to the secular equation. The details of this calculation can be found in the original manuscript (10). The resulting juxtaposition of atates 18 shown in Fig. 3. The $2 \pi_{3 / 2}$ and $2 \Sigma_{1 / 2}^{+}$states are separated by only $-0.08 \mathrm{eV}$, while $2 \pi_{1 / 2}$ is separated from the mean of the other states by $-0.95 \mathrm{eV}$. The experimental data (Fig. 1) reveal two peaks, the lower one more Intense, and separated from the upper one by about $0.92 \mathrm{eV}$. This splitting $1 \mathrm{~s}$ very nearly that of the ledine atom, not $2 / 3$ of this value as in HI. This 18 because the lodine in CsI 18 almost spherically symuetric (1.e. the $\pi-\sigma$ splitEing is small compared to the spin-orbit splitting). The more Ionic alkali halides, which have the smallest $\pi m \sigma$ splittings; have resultant splittings characteristic of individual, apherically symetric lons only slightly perturbed by the neighboring ion.

Taking inte account the peak widths, the corresponding calculation for Cabr predicts a partial aplitting into twio peaks, with a separation of $-0.4 \mathrm{eV}$, and for CaC1 and CaP a single peak, in good agreement with the experimental results (10). Predictions regardinf this fine structure for the other alkali halldes can be found in the original article (10).

A rewarding aspect of photoelectron spectroscople investigations is that a spectrum of a single wolecular systew, when finally understood, enables one to predict the behavior of the entire howologous series. This is, of course, a consequence of the wolecular orbital etructure of matter. One can observe this in the

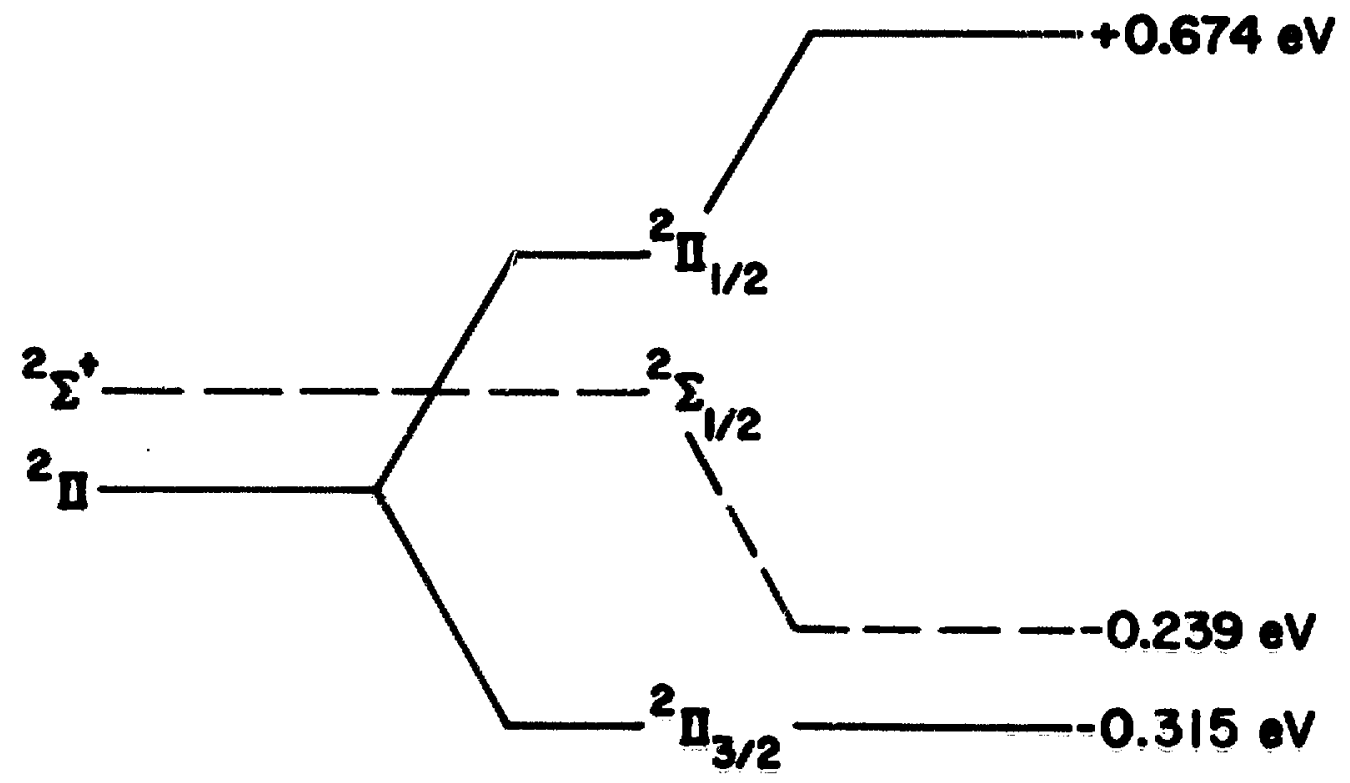

Fig. 3. Final eplitting diagram of CoI, including în interaction. 
existing spectra of $\mathrm{HF}, \mathrm{HCl}, \mathrm{HBr}$ and $\mathrm{HI}$; the corresponding diatomic halogens; the Group III monohalides of $\mathrm{Tl}, \mathrm{In}, \mathrm{Ga}, \mathrm{Al}$ and $\mathrm{B}$; the Group II dihalides, and also the alkali halides. As our knowledge of the Ionic states of small molecules increases, it becomes attractive to predict their uss spectra. Table 1 is a composite of parent to fragment $\left(\mathrm{MX}^{+} / \mathrm{M}^{+}\right)$ratios of the diatomic alkali halides, primarily obtained from electron impact data. One can see a strong correlation with the ionicity of the molecule, the most Ionic (CsF) having an undetectable parent ion, whereas the least Ionic (LII) has almost an order of magnitude more parent than fragment. We can rationalize these observations from the photoelectron spectroscopic analysis by first noting that $70 \mathrm{eV}$ electron impact Ionization will be dominated by ionization from the lower lying halogen-1ike orbitals. To first order, the magnitude of the $\pi \rightarrow 0$ splitting will determine whether at least one of the fine structure states of this p-like orbital is bound. When the $\pi-\sigma$ splitting is omall, both the $2 \pi$ and $2 \Sigma$ Ionic states tend to be repulsive, and hence strongly favor the formation of fragment lons. When the $\pi-0$ splitting is celatively large, as in the cases of LIC1, LIBr and LII, the $2 \pi$ state becomes significantly bound, and provides a

\section{TABLE 1}

Parent to fragment ratio $\left(\mathrm{MX}^{+} / \mathrm{M}^{+}\right)$in the Ionization of alkali halides

\begin{tabular}{llllll}
\hline & \multicolumn{1}{c}{ F } & C. & Br & I & Refs. \\
\hline L1 & $0.11-0.31$ & 3.39 & 2.18 & 7.46 & (a) \\
Na & 0.026 & 0.59 & 0.71 & 1.17 & (b) \\
K & 0.002 & 0.17 & 0.29 & 0.56 & (b) \\
Rb & & 0.07 & & & (b) \\
& & 0.06 & 0.155 & 0.24 & (c) \\
Cs & & 0.012 & & & (b) \\
& 0.00 & 0.014 & 0.07 & 0.26 & (d) \\
& $<.005$ & 0.015 & 0.028 & 0.14 & (c) \\
\hline
\end{tabular}

(a) (Electron impact), J. Berkowitz, H. A. Tasana and W. A. Chupka, J. Chen. Phys. 36, 2170 (1962).

(b) (Electron inpact), J. Berkowitz and W. A. Chupke, J. Chen. Phys. 29, 653 (1958).

(c) (Photon impect), J. Berkow1tz, J. Chem. Phys. 50, 3503 (1969);

J. Berkowitz, Adv. High Temp. Chem. 3, 123 (1971).

(d) (Electron Impact), P. A. Akishin, L. K. Gorokhov and L.' N. Sidorov, Dok1. Akad. Nauk SSSR 135, 113 (1960). 
source of parent ion. By including the effects of spin-orbit splitting, it is even possible to rationalize the nonmonotonicity in the fragmentation behavior of LIC.1, LIBr and LII.

So far, we have given examples of other types of molecules which can be examined by $584 \AA$ photoelectron spectroscopy. The Information obtained is still primarily of a chemical nature. We have not yet given any evidence to support our thesis that future studies will prove rewarding to atomic and chemical physicists. We oha11 postpone this denonstration a bit longer to consider briefly the parallel developwent of photoelectron spectroscopy in the $x$-ray region that has been pioneered by Prof. Siegbahn and his colleagues at Uppara (11).

The photolonization cross sections of molecules, which are near their maximum at $584, \bar{k}$, are typically -two orders of magni-

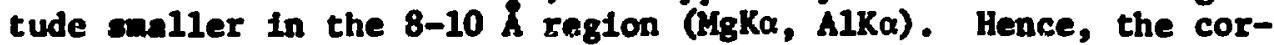
responding photoelectron spectroscopic messurenents on free wolecules are considerably wore difficult at this photon energy. The partial cross sections for lonization from the valence shell. decrease even wore drastically, and represent a onall fraction of the total ionization in the $x$-ray region. By comparison with the wealth of data accumulated at $584, \lambda$, relatively fewer molecular syeter have been studied at $9.9 \AA$, and most of these studies have been confined to the larger partial cross sections of the inner she11s. These inner shells are locslized strongly in the vicinity of the atom that ake up a particular wolecule. The photoelectron epectra of these inner she11s are, to zeroth order, atonic photoelectron spectra. However, these atomic lines are shifted frum their free aton esergy valuas by the chentcal environent in which they reaide. This chenteal shift, typically a few volts, can be menured ascurately and has been extensively correlsted with the charge distribution within the molecule, and the valence state of particular atoms in the wolecule. The only point we wish to anke here is that this branch of the photoelectron spectroscopy of wolecules has also been strongly oriented toward the deternination of chemical Information. Indeed, the acronym ESCA (Blectron Spectroscopy for Chentcel Analyaid is an indication of the prinary thrust of this research. X-ray photoelectron spectroscopy has also been extended recently to the study of high temperature species (12), where the combined requirenents of working with molecular bean and with low photolonization cross sections anes the experiment. very difficult.

\section{PHYSICAL STIDIES}

The properties that I wish to characterize here as phyelcal ones are intennities and angular dietributions, from wich one can Infer transition probabilities and phase shifts. Thees quantities 
are determined by the nature of the continuum wave function, as well as that of the neutral ground state, whereas by and large, chemical properties are discussed in terms of the initial state's molecular orbitals.

In the dipole approximation, which is excellent for low-energy photons, the differential photoionization cross section for unpolarized incident radiation is

$$
\frac{d \sigma(\varepsilon)}{d \Omega}=\frac{\sigma_{t}(\varepsilon)}{4 \pi}\left[1-\frac{\beta(\varepsilon)}{2} P_{2}(\cos \theta)\right]
$$

where $\varepsilon$ is the energy of the ejected photoelectron, $\sigma_{t}(\varepsilon)$ is the total photolonization cross section, $\theta$ is the angle between the incoming photon direction and the outgoing electron direction, $P_{2}(\cos \theta)=1 / 2\left(3 \cos ^{2} \theta-1\right)$, and $B(\varepsilon)$ is an aaymmetry parameter which, in the approximation of Russel1-Saunders coupling and oneelectron wave functions can be shown to take the form

$B(E)=\frac{\ell(\ell-1) R_{\ell-1}^{2}(E)+(\ell+1)(\ell+2) R_{\ell+1}^{2}(c)-6 \ell(\ell+1) R_{\ell-1} R_{\ell+1} \cos \left[\delta_{\ell+1}^{(l)}-\delta(l)\right.}{(2 \ell+1)\left[\ell R_{\ell-1}^{2}(l)+(\ell+1) R_{\ell+1}^{2}(\varepsilon)\right]}$

In this latter expression, $R_{\ell \pm 1}(\varepsilon)$ is the dipole matrix element, defined by

$$
R_{\ell \pm 1}(\varepsilon)=\int_{0}^{\infty} P_{n \ell}(r) r P_{\varepsilon, \ell \pm 1}(r) d r
$$

and $\delta_{\ell \pm 1}(\varepsilon)$ are the phase shifts of the continuum $l \pm 1$ partial waves with respect to free waves.

Even without detailed calculations, these expressions can teach us something about experimental design and the nature of the photoionization process. From eq. (1) we note that when $P_{2}(\cos \theta)=0$, the differential cross section will be independent of $B$. This occurs for $\theta=54^{\circ} 44^{\prime}$. From the wealth of photoelectron spectra currently in the literature, extremely few measurements have been made at this angle. Hence, in general, the relative intensities of the various bands, which have been primarily measured at $\theta=90^{\circ}$, are not meaningful unless one has an Independent measurenent of $B$ at that energy. However, if one can perform measurenents at the $54^{\circ} 4^{\prime}$ angle, relative Intensities can be directly related to calculated intensities without requiring a knowledge of $B$.

The electron energy analyzer which was previously mentioned as a high transmission device for studies of molecular beams, can 
conveniently be constructed with $\theta$ near the $54^{\circ} 44^{\circ}$ value. The design parameters of the particular analyzer constructed at Argonne (9) happen to optimize at $\theta=60^{\circ}$. In addition, it has no retarding, accelerating or focusing lenses. Taking into account the $\triangle E / E$ effect (13), this analyzer appears to have a flat transmission function for electrons with kinetic energy greater than $-1.5 \mathrm{eV}$, falling off to lower energy.

I shall describe below three separate experiments which involved accurate relative intensity measurements with this instrument, and show how an interpretation of the results by appropriate theory has given us new insight into the phototonization process.

A. Comparison of photoelectron intensities and Franck-Condon factors in the photoionization of $H_{2}, \mathrm{HD}$ and $\mathrm{D}_{2}$.

The relevant transitions in the $584 \AA$ photoelectron spectrum of $\mathrm{H}_{2}$ are

$$
\mathrm{H}_{2}\left(\mathrm{X}^{1} \Sigma_{\mathrm{B}}^{+}, \mathrm{v}^{\prime \prime}=0\right)+\mathrm{h}_{\mathrm{v}+\mathrm{H}_{2}^{+}}\left(\mathrm{X}^{2} \Sigma_{\mathrm{g}}^{+}, \mathrm{v}^{\prime}=0,1,2 \ldots\right)+\mathrm{e}^{-}
$$

Designating the Initial state as $a$, and the final state as $b$, we may formaliy write the cross section for this process as

$$
\sigma(a \rightarrow b)=\frac{8 \pi^{3} e^{2} v}{c} \sum_{b} \sum_{\alpha}|<b| M_{\alpha}|a>|^{2}
$$

where $v$ is the frequency of incident radiation, $e$ and $c$ have their usual meaning and $\alpha$ is the index for electron coordinates $x, y$ and z. The first summation is over the degenerate final states.

In the Born-Oppenheimer approximation, both initial and final states are written as products of electronic functions $\psi$, vibrational functions $P$ and rotational functions $Y, i . e$.

$$
\begin{aligned}
& |a\rangle=\psi_{a}\left(R, \underline{r_{1}}, \underline{r_{2}}\right) R^{-1} P_{a}\left(v_{a}, R\right) Y\left(J_{a}, m_{a}\right) \\
& \langle b|=\psi_{b}^{*}\left(R, \underline{r_{1}}, \underline{r_{2}}, E_{e}\right) \dot{R}^{-1} P_{b}\left(v_{b}, R\right) Y *\left(J_{b}, m_{b}\right)
\end{aligned}
$$

where $R$ is the internuclear distance, $r_{1}$ and $r_{2}$ are the coordinates of the two electrons with respect to axes fixed in the molecule, $v$ is the vibrational quantum number and $J$ and $M$ are the rotational quantum number and its projection, and $E_{e}$ is the electron kinetic energy. If for the moment we assume the rotational functions to be uncoupled and integrate over the rotational coordinates, we san write the transition matrix element as follows: 


$$
\left\langle\mathbf{b}\left|\mathbf{M}_{\alpha}\right| \mathbf{a}\right\rangle=\int_{0}^{\infty} \mathrm{P}_{b}^{\star M_{\alpha}} \mathbf{P}_{\mathbf{a}} \mathrm{dr}
$$

where

$$
M_{\alpha}=\iint \psi_{b}^{*}\left(r_{1 \alpha}+r_{2 \alpha}\right) \psi_{a} \underline{d r_{1}} \underline{d r_{2}}
$$

Since $\psi_{b}$ and $\psi_{a}$ are functions not only of the electron coordinates but also of the internuclear distance $R$, it follows that $M_{\alpha}$ will also be a function of $R$. In addition, $\psi_{b}$ is a function of $E_{e}$, and hence $M_{\alpha}$ must also be dependent on $E_{e}$. In calculating Franck-Condon factors and identifying them with vibrational intensities, one is explicitly ignoring this dependence, and is writing eq. 6 in the form

$$
\left\langle b\left|M_{\alpha}\right| a\right\rangle=M_{\alpha}^{(0)} \int_{0}^{\infty} P_{b}^{*} P_{a} d R
$$

where the integral corresponds to the Franck-Condon factor, and $M_{\alpha}(0)$ is the value of $M_{\alpha}$ at a typical $R$ - customarily chosen to be the equilibrium distance in the electronic ground state $a$. Flannery and Öpik (14) have shown how $M_{\alpha}$ varies with $R$ and $E_{e}$ in the fonization of $\mathrm{H}_{2}$, choosing one reasonable model for $\psi_{b}$ and $\psi_{a}$. Itikawa (15) has expanded both upon this model and upon a more sophisticated one for $\psi_{b}$. In each case, he has expressed the resulting $M_{\alpha}$ function as a power series

$$
M_{\alpha}=\gamma+\delta\left(R-R_{e}\right)+\varepsilon\left(R-R_{e}\right)^{2}
$$

(in which $\gamma, \delta$ and $\varepsilon$ are constant), inserted it into eq. 6 , and calculated the vibrational intensities.

Experimentally, we (16) have carefully integrated the areas under the vibrational bands obtained in the 584 \& photoelectron spectrum for $H_{2}, H D$ and $D_{2}$. The experiments were performed with a resolution width of $20 \mathrm{meV}$, where rotational structure was beginning to influence the peak shape. The relative intensity was taken as the area divided by the corresponding electron energy. They were plotted as ratios of the corresponding Franck-Condon factors vs. vibrational number, where the Franck-Condon factors are very precise calculations by Peek (17). The results for $\mathrm{D}_{2}$ are shown in Fig. 4, together with data by Frost et al. (18) and Itikawa's calculations. If the approximations involved in eq. (8) were valid, we would expect perfect agreement with Franck-Condon factors, in which case the data points should describe a straight horizontal line. Clearly, the data points have a positive slope, Indicating a substantial deviation from purely Franck-Condon behavior. The agreement with Itikawa's case (C) is particularly 


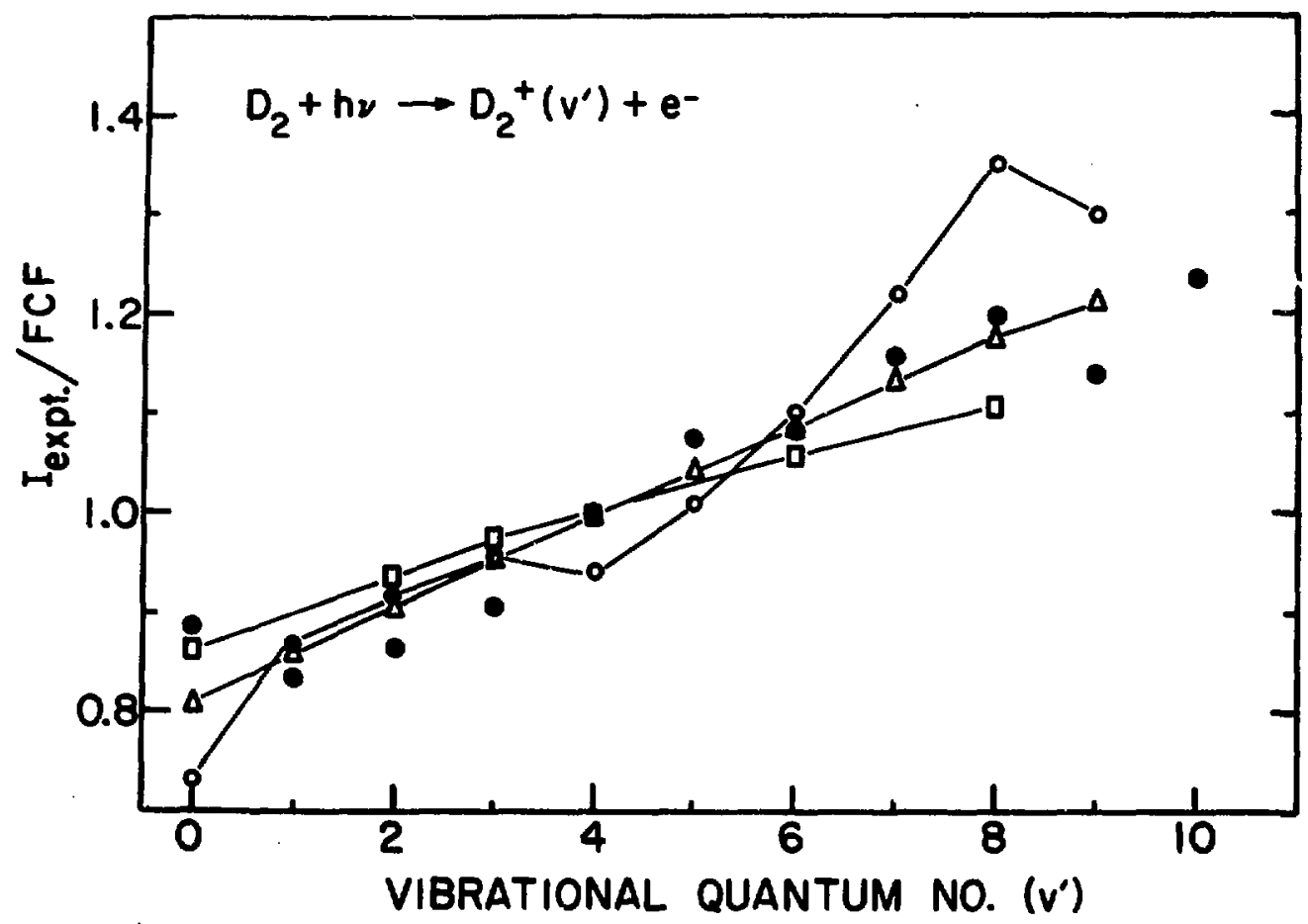

Fig. 4. The ratio of the experimental (or calculated) vibrational intensity to the Franck-Condon factor for $D_{2}$, plotted as a function of the vibrational quantum number $v^{\prime}$. O O Frost, McDowel1 and Vroom; $\square \square$ Itikawa, case (B); $\Delta \Delta$ Itlkawa, case $(C)$; and $\bigcirc$ Berkowitz and Spohr.

good, indicating that this particular choice of continuum function is a reasonable one, and also providing support for the view that the essence of the physics has been included in the calculations, despite several approximations in Itikawa's calculations.

B. Rotational band shapes in the photoelectron spectra of $\mathrm{HF}$ and $D F$

It has long been realized that the peak shapes observed in photoelectron spectroscopy contain useful information. When individual vibrational levels are not resolved, it is assumed that a broad peak corresponds to a considerable change of geometry on lonization, while a narrow peak suggests that the ion has a similar geometry to that of the neutral molecule. However, much less work has been concerned with the effects of rotational broadening which may be investigated when Individual vibrational levels of the ion are resolved. 
The photoionization process in HF corresponding to

$$
\mathrm{HF}\left({ }^{1} \Sigma^{+}, \mathrm{v}^{\prime \prime}=0\right)+\mathrm{hv} \rightarrow \mathrm{HF}^{+}\left({ }^{2} \pi_{1 / 2},{ }^{2} \pi_{3 / 2}, \mathrm{v}^{\prime}=0,1,2, \ldots\right)+\mathrm{e}^{-}
$$

is of particular interest in this context, because the $2 \pi / / 2$ and

$2 \pi_{3 / 2}$ states are incompletely resolved, and the shape of an individual vibrational band is determined not only by the nature of the rotational transitions, but also by the spin-orbit coupling. By considering the hamiltonian of the entire system, calculating the rotational line strength of each rotational transition and using an experimental line width derived from a nearby atomic line obtained in the same experiment, a theoretical band contour can be constructed. In this instance, the theoretical band contour was calculated for several assumed values of the spin-orbit coupling constant. The best fit enabled us to extract a rather accurate value of the spin-orbit coupling constant from the band contour.

We shall only sketch the outlines of the calculation here. For details see ref. (19).

If we only consider Ionization of the outermost $(\pi)^{4}$ system of HF into the $s$ partial wave, the final state of the system ion plus electron has the configuration $\pi^{3} \sigma$, which gives rise to ${ }^{3} \pi$ and $l_{I}$ states. These two states are degenerate, 1.e. the triplet and singlet Rydberg series approach the same fonic limit. The ${ }^{3}$ I state consists of three components with $\Omega=2,1$ and 0 . The ${ }^{1}$ II state has $\Omega=1$. In the nonrotating molecule, electric dipole transitions from the ground state of the neutral molecule $\left({ }^{1} \Sigma^{+}\right)$ are only allowed to the $l_{\Pi_{1}}$ state. However, spin-orbit coupling mixes the ${ }^{l_{\Pi}}$ and ${ }^{3} \Pi_{1}$ states, and the rotation of the molecule couples the three components of the ${ }^{3} \pi$ state by means of spinuncoupling (20). Hence, transitions become allowed to all four final states. The hamiltonian describing the interactions is of the form

$H=B\left[\left(J_{x}-L_{x}-S_{x}\right)^{2}+\left(J_{y}-L_{y}-S_{y}\right)^{2}\right]+H^{S O}=H^{R}+H^{S O}$

where $J$ is the total angular momentum, $L$ and $S$ are the electronic orbital and spin angular momenta, and $H^{\text {SO }}$ is the spin-orbit contribution. This is written in matrix form in Table 2 .

This matrix is then diagonalized, and the rotational line strengths are calculated by using the Hön1-London factors for a $1_{\Sigma} \rightarrow I_{I}$ transition (21), which are
R branch intensity $\propto(\mathrm{J}+2)$
$Q$ branch intensity $\propto(2 \mathrm{~J}+1)$
$P$ branch intensity $\propto(J-1)$ 
TABLE 2

Matrix elements of $H=H^{R}+H^{80}$ for ${ }^{3} \Pi$ and ${ }^{1} \Pi$ states in Hund's case (a)

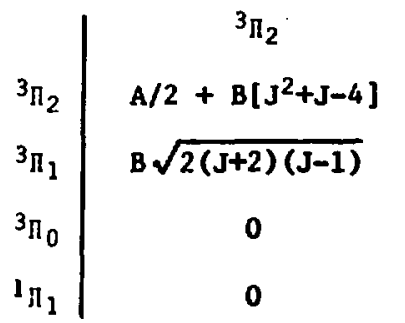

$B \sqrt{2(J+2)(J-1)}$
$B J(J+1)$
$B \sqrt{2 J(+1)}$
$A / 2$

$3 \Pi_{0}$
0
$8 \sqrt{2 J(J+1)}$
$-A / 2+B J(J+1)$
0

$I_{\|_{l}}$
0
$A / 2$
0
$B\left[J^{2}+J-2\right]$

${ }^{1} A$ is the spin-orbit coupling for the lon ${ }^{2} \|$ state.

The vibrational band contour is then constructed from the sum of rotational lines, each one broadened by an experinental line width and weighted with the appropriate intensity and Boltzmann factor. The only variable parameters in this approach are the spin-orbit coupling constant and the lonization potential, which we define as the difference in energy between the $J=0$ rotational level of the molecule and the $J=3 / 2$ rotational level of the ${ }^{2} \pi_{3 / 2}$ state of the ion. Only the spin-orbit coupling constant affects the band shape.

Figure 5 shows the experimental results for DFt, $v^{\prime}=0$, together with the Ar+ ${ }^{2} \mathrm{P}_{y / 2}$ line and the calculated contour that $\mathrm{g}^{\mathrm{ive}}$ the best fit. From this fitting procedure, we have deduced a spinorbit coupling constant of $290 \pm 10 \mathrm{~cm}^{-1}$. This is significantly different from the value $240 \mathrm{~cm}^{-1}$ that had been deduced by weasuring the partially resolved splitting in the photoelectron spectrum. It 18 also in line with the ratio of doublet splittings in HXt and the corresponding $X, g^{i v e n}$ in Table 3 . For the determination of Ionization potentials, rapid scans are preferable since they minimize the likelihood of any change of surface potential occurring between the argon peaks (used for calibration) and the $\mathrm{HF}^{+}$or $\mathrm{DF}^{+}$peaks. The values of the ionization potentials obtained from the best fit between calculation and experiment are

$$
\begin{aligned}
& \text { HF: } 16.044 \pm 0.003 \mathrm{eV} \\
& \text { DF: } 16.058 \pm 0.003 \mathrm{eV}
\end{aligned}
$$

These errors are an order of magnitude smaller than the experimental line width. After correcting for molecular and ionic zero point energies, there is a residual discrepancy which way be due to an electronic isotope effect $(19,22)$.

The ionization potentials given above are slightly different from those obtained previously by photolonization mass epectrometry (23). This is due to the difficulty of choosing a proper 


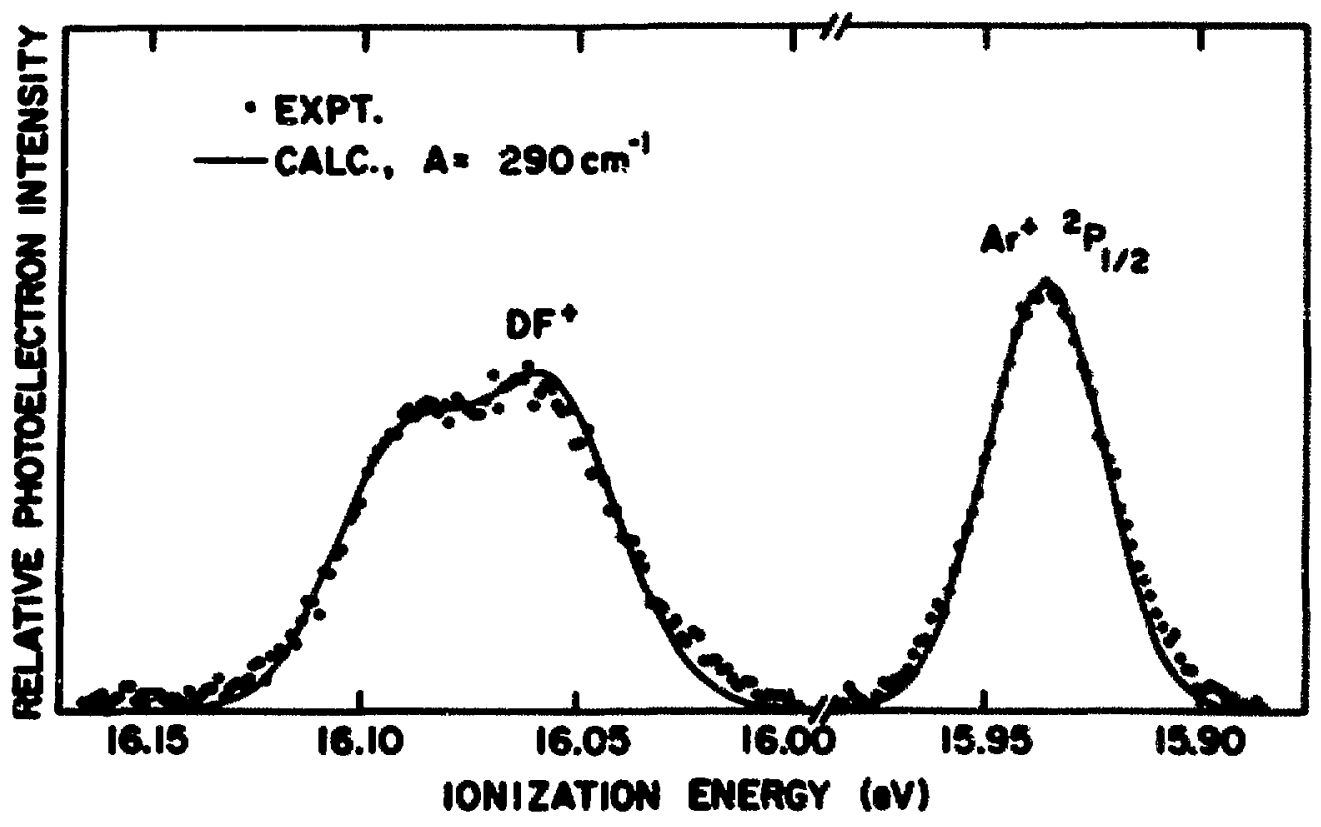

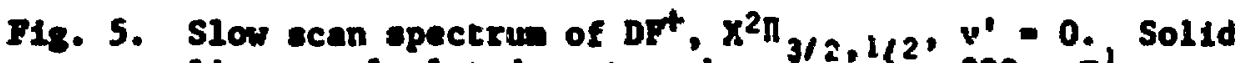
line: calculated contour based oñ ${ }^{2}=290 \mathrm{~cm}^{-1}$. - experimental points.

TABLE 3

Ratio of wolecular to atoaic spin-orbit splitting

\begin{tabular}{|c|c|c|c|}
\hline Ion & $\begin{array}{c}2 \pi_{1 / 2^{-2} \pi_{3 / 2}} \\
c \pi^{-1}\end{array}$ & ${ }^{{ }_{C} P_{1 / 2}{ }^{-2} P_{1 / 2}}$ & $\operatorname{Rat} 10$ \\
\hline $\mathrm{HI}^{+}$ & 5650 & 7603 & 0.743 \\
\hline $\mathrm{HBr}^{+}$ & 2653 & 3685 & 0.720 \\
\hline $\mathrm{HCl}^{+}$ & 643 & 881 & 0.730 \\
\hline hrt & 290 & 404 & 0.72 \\
\hline
\end{tabular}

threshold function for the mass spectrometric deternination, as well as the less detalled treatinent of rotation. The results given here wust be considered nore precise.

Although only the $s$ wave has been considered in the theory given here, we have reason to believe (19) that the inclusion of the doninant d wave will not seriously affect our conclusions. The d wave will allow larger angular womentum transfers to take place 
chan for an s wave and tranattions becone allowed from HF (J) to $H \mathrm{~F}^{+}(\mathrm{J} \pm 1 / 2, J \pm 3 / 2, J \pm 5 / 2)$. The effect of thase extra transitions will be to broaden the overall peake with respect to that calculated for waves only, and to reduce any dip between the two halves of the doublet.

It should be noted that this analysis has presupposed an experinent conducted at $\theta=54^{\circ} 44^{\circ}$. If the experinent were conducted at very different $\theta$, such se $90^{\circ}$, the angular distribution of each rotational trasition at the energy of the experiment would have to be known.

C. Spin-orbit conpling and the ratio of photolonization cross sections in sone atoms.

One of the earliest experfinents reported in photoelectron spectroscopy corresponded to the reaction

$$
\begin{aligned}
& \text { Ar } \quad A x^{+}\left({ }^{2} P_{3 / 2},{ }^{2} P_{1 / 2}\right)+e^{-} \\
& k r\}+h v+k r+\left({ }^{2} p_{3 / 2},{ }^{2} p_{1 / 2}\right)+e^{-} \\
& x e \sqrt{x e+\left({ }^{2} P_{3 / 2} \cdot{ }^{2} P_{1 / 2}\right)+e^{-}}
\end{aligned}
$$

When easurements becowe a bit wore refined, it was found that the ratios of intenoities of the ${ }^{2} p_{3 / 2}$ to ${ }^{2} P_{1 / 2}$ were 1 ess than $2: 1$ (their ratio of statistical weights) in every inatance. In 1968 , Sanson and Calrns (24) reported this ratio to be $1.98,1.79$ and 1.60 for $A x, K r$ and $X e$, respectively. We have recently reneasured these ratios with our cylindrical onalyzer, and find fair agreement.

In addition, we (25) have wasured the ratio of spin-orbit split states formed in the Ionization of the set $\mathrm{Zn}$, Cd and $\mathrm{Hg}$.

Thus

$$
\begin{aligned}
& 2 n \text {... (3d) }{ }^{10}(4 s)^{2} \quad 2 n^{+}\left({ }^{2} D_{5,2},{ }^{2} D_{3 / 2}\right)+e^{-} \\
& \text {Cd...(4d) }{ }^{10}(5 e)^{2}+h v \rightarrow \operatorname{Cd}^{+}\left({ }^{2} D_{5 / 2,}{ }^{2} D_{3 / 2}\right)+e^{-} \\
& \text {Hg...(Sd) }{ }^{10}(6 s)^{2} \quad \mathrm{Hg}^{+}\left({ }^{\prime} \mathrm{D}_{5 / 2},{ }^{2} \mathrm{D}_{3 / 2}\right)+\mathrm{e}^{-}
\end{aligned}
$$

The ratio of statistical weights in this case should be 1.50 . For $\mathrm{zn}^{+}$it appears to be close to this value, but for $\mathrm{Cd}^{+}$it is 1.79, and for $\mathrm{Hg}, 2.18,1 . e$. larger than statistical.

In an attempt to rationalize these observations, Dr. Walker (25) has recently performed sone calculations using Dirac-siater relativistic wave functions for both the bound and continuin atates. From these calculations has energed a general 
understanding of branchir.g ratios and their departure from atatistical weight predictions, which is a generalization of Cooper's calculations (26). Cooper had previously noted that, although the dipole selection rules permitted $\Delta l= \pm 1$, the $\ell+1$ channel usually dominates the crose section.

At very low photoelectron energies, the first major maximum of the continum orbital of the $\ell+1$ channel will 11 outside the region of space occupied by the bound orbital. As the photoelectron energy Increases, this maximum noves nearer the nucleus, and the dipole ratrix element increases. When the photoelectron energy increases still further, the first major naximum of the continuum orbital begins to overlap the nodes of the bound orbital (if any) and the dipole atrix element decreases and finally changes sign, giving rise to the Cooper minimu in the partial crose section.

Now consider what happens when spin-orbit coupling is introduced. This sauses the $f=l-1 / 2$ component of the bound orbital, for which the spin-orbit potential is attractive, to be drawn olightly closer to the nucleus than the $j=h+1 / 2$. As a result, when the photoelectron energy Increases from zero, the continuum orbital will have a greater overlap with the $f=l+1 / 2$ component of the bound state, and the ratio $\sigma(l+1 / 2) / \sigma(l-1 / 2)$ will be greater than the statistical value of $(l+1) / \ell$. Similarly, the continuum orbital will overlap the first node of the $j=l+1 / 2$ before that of the $f=l-1 / 2$, and in this part of the spectrum $\sigma(l+1 / 2) /$ $o(l-1 / 2)<(l+1) / \ell$. Figure 6111 ustrates this. The same considerations hold after the Cooper minimum is passed. Thus we can make the generalization that if the partial cross section is rising, the ratio of cross sections 18 greater than statistical, while if the partial cross section 18 falling, the ratio will be less than statistical. For the rare gases $\mathrm{Ar}, \mathrm{Kr}$ and $\mathrm{Xe}$, the cross section is falling at $21.2 \mathrm{eV}$, while for $\mathrm{Zn}, \mathrm{Cd}$ and $\mathrm{Hg}$ it is rising.

\section{SUMMARY AND CONCLUSIONS}

There are other physical aspects of photoelectron spectroscopy, such as the measurement of the angular distribution asymmetry parameter $B$ as a function of photoelectron energy, and the determination of branching ratios and $\beta^{\prime} s$ across autoionizing peaks for which there is neither time nor space at this conference. However, I hope that $I$ have given enough examples to support the view that the branching ratio of photoelectron spectroscopy between chemistry and physics will move more strongly to the latter in the next few years. 


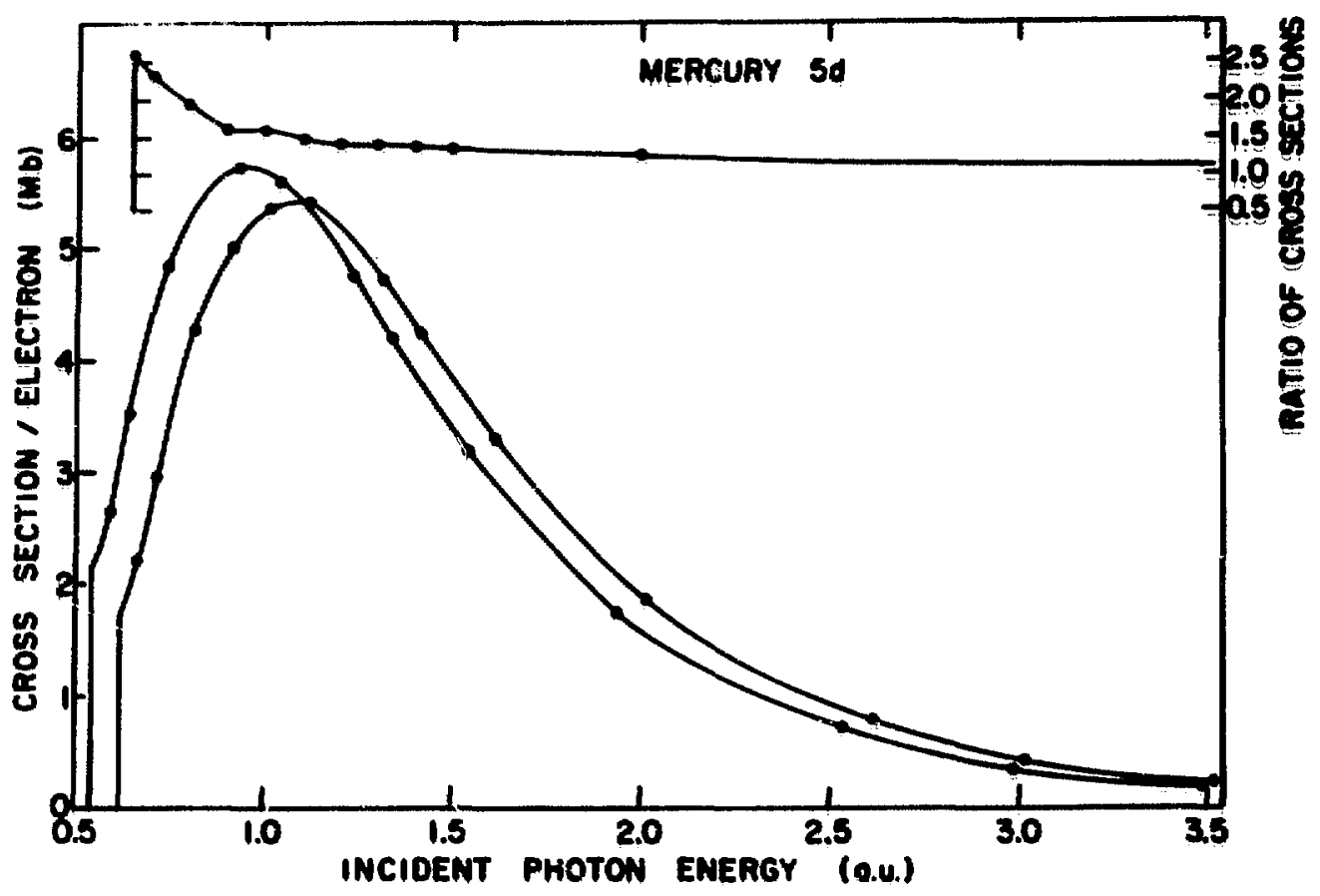

Fig. 6. Cross section per electron for photoionization of the $5 d_{5,2,3 / 2}$ orbital of mrcury as a function of incident photon energy. 5d $\mathrm{d}_{3 / 2}$ - open circles and $5 \mathrm{~d}_{5 / 2}$ - closed circles. The topaost curve shows $\sigma_{5 / 2} / \sigma_{3 / 2}$ as a function of Incident photon energy.

\section{REFERENCES}

*Work performed under the auspices of the U.S. Atonic Energy Comission.

1. F. I. V1lesov, B. L. Kurbatov and A. N. I'erenin, Dok1. Akad. Nauk SSSR 138, 1329 (1961); Soviet Physics Doklady 6, 490 (1961).

2. D. W. Turner and M. I. Al Jobory, J. Chem. Phys, 37, 3007 (1962).

3. J. Berkowitz, to be published.

4. D. W. Turner, C. Baker, A. D. Baker and C. R. Brundle, "Molecular Photoelectron Spectroscopy," Wiley-Interscience, London (1970). 
5. As an atd to the reader in locating some of the yoluninous licereture on photoslectron spectroscupy of molecules at $584 \mathrm{~A}$, the fournals in which recent contributions by the aforemonfloned authors appent are listed below:

W. Beck. 3. Waturf : H, Bock, A. Schwets, W. Schaeffer, Anges, Chese Int Ed, $\mathrm{T}$. A. Carlson, J, Coll1n,

J. B1ec. Spectr:i D. C. Frost and C. A. McDowall, Chen.

Phye, Letters: E, Het3bronner, Helv. Ohtm. Acta;

D. R. Lojd, A, E, Orchard, J. Chem, Sor. Par. Trans. 2;

E. Lindholin, Phyece scripta; $W$. C. Price, Proc. Roy.

Soc. and $\mathrm{J}$, Phys. B.

6. A. Jophthon, A. Morris, D. J. Sntch and X. J. Rose, Chen. Phos. Latters I, 497 (1970); H. Jopathan, D. J. Sulth and K. J. Rose, J. Chen, Ehys, 53, 3758 (1970); H. Jonthan. A. Worrte, k. J. Roes and D. J. snteh, J. Chem. Phys. sh. 4954 (1971): L. Golob, H. Jonethan, A, Horrts, H. Okuda and X. J. Rose, J, Blec, Spec. 1, 506 (1973).

7. D. C. Iroet, S. T. Lee and C. A. Meboure11, Chen. Phye. Letters 17, 153 (1972); A. B. Cornford, D. C. Frost. F. G. Herring and C. A. Hepowe11, J. Chen. Phye. 54, 1872 (1971); D. C. Frost, S. T. Lee and C. A. Mebonell, "Iontsation Botentiels of Short-11ved Species," presented at 2lst Annual Conf. on Mnes Spectrometry and Alled Topics, San Francleco, Cellf. . Yay 20-25, 1973.

8. H. U. Eroto and R. J. Suffolk, Chem Phys, Latters 17, 213 (1972).

9. J. Berkoutte, J. Chen. Phys. 56, 2766 (1972); J, Barkowtiz and J. L. Dehor, J. Chen. Phje. 51, 3194, (1972).

10. J. Berkowits, J. I. Dehmer and T. E. H. Walker, "PES of High Temperature Vapore IV. The Cestur Halides," J. Chen. Phys. (acceptad for publication).

11. See, for exnmple, "Bsch - Atonic, Holecular and Solid State Structure Studied by Heane of Electron Spectroscopy,"

K. Siegbehn and colleggues, Almquist and Wiksells, publishers, Uppeale (1967); "BSCA Applied to Iree Kolecules," K. Slegbahn and colleagues, N. Holland Publ. Co., Ansterdan (1969).

12. Y. S. Whodeev, H. Siegbahn, K. Hanrin, K. Siegbahn, Chea. Phye. Lett. 19, 16 (1973).

13. J. Berkowtic and P. H. Guyon, Int. J. Mass Spec. Ion Phys. 6, 301 (1971).

14. M. R. Flannery and U. ÖpIk, Proc. Phys. Soc. (London) 86, 491 (1965).

15. Y. Itikaws, J. Electr. Spectr, and Related Phen., In press. 
16. J. Berkowitz and R. Spohr, J, Electr. Spectr, and Related Phen., In press.

17. J, M. Peek, private commication.

18. D. C. Frost, C. A, MeDowell and D. A. Vroom, Proc. Ray, Soc, (London) A296, 566 (1967).

19. T. E. H. Walker, P. M. Deherer and J. Barkowtts, "Rotational Band Shapes in Photoelectron Spectroscopy: HF and DF,"

J. Chen, Phys. (nubitited for publication).

20. G. Herzberg, "Holecular Spectra and Holecular Structure. II. Spectra of Diatonic Holecules," D. Van Mostrand, Princeton, N.J. (1950).

21. J. T. Hougen, "The Calculation of Rotational Energy Levels and Rotational Line Intenafties in Diatomic Holecules," MBS Monosraph 115 (1970).

22. F. R. Bunker, J. Kol. Spec. 28, 422 (1968).

23. J. Bexkowttz, W. A. Chupkn, P. H. Guyon, J. H. Holloway and R. Spohr, J. Chem. Phyn. 5h, 5165 (1971).

24. J. A. R. Samson and R. B. Calrna, Phys, Rev. 173, 80 (1968).

25. T. B. H. Walker, J. Berkowitz, J. L. Dehmer and J. T. Waber, "Ratios of Photolonization Crose Sections of Spin-0rbit Split States," Phys. Rev. Lett. 31, 678 (1973).

26. J. W. Cooper, Phys, Rev. 128, 681 (1962). 